\title{
Was Patienten zufrieden macht
}

\author{
PATIENTENZUFRIEDENHEIT TEIL 1 Zufriedene Patienten kommen wieder und \\ empfehlen ihren Therapeuten weiter. Der Stellenwert der Patientenzufriedenheit \\ wird von vielen Einrichtungen zwar als Erfolgsfaktor erkannt, wird jedoch selten \\ professionell erfasst. Karen Friedel hat für ihre Bachelorarbeit zu diesem Thema \\ recherchiert und fasst hier die Theorie zusammen. In der nächsten Ausgabe finden \\ Sie ein praktisches Beispiel, wie man die Zufriedenheit schriftlich evaluieren kann.
}

m Prinzip strebt jeder Physiotherapeut danach, gute Arbeit zu leisten. Aber was ist unter dem Begriff „gut“ zu verstehen? Wie unterscheidet man einen guten von einem weniger guten Therapeuten? Diese Frage stellt sich, wer sich Gedanken um die Qualität und Effektivität seiner Behandlungen macht.

Zudem ist die Erbringung qualitativ hochwertiger Dienstleistung nicht freiwillig: Sie ist gefordert, und zwar von Seiten des Gesetzgebers (॰ Kasten „Auszug aus dem SGB V“).

\section{Messung der Patientenzufriedenheit ist Teil des Qualitätsnachweises > Das Thema Qualität ist also längst Thema in der Phy- inzwischen zertifizieren, um eine offizielle Bestätigung ihrer Qualität vorweisen zu können.} siotherapie. Und da die Kosten im Gesundheitssystem permanent steigen, wird der Gesetzgeber bzw. die Krankenkasse künftig vorrangig diejenigen Behandlungen bezahlen, bei denen ein wissenschaftlicher

\section{AUSZUG AUS DEM SGB V}

\section{$\S 135 a:$ Verpflichtung zur Qualitätssicherung}

„Die Leistungserbringer sind zur Sicherung und Weiterentwicklung der Qualität der von ihnen erbrachten Leistungen verpflichtet. Die Leistungen müssen dem jeweiligen Stand der wissenschaftlichen Erkenntnisse entsprechen und in der fachlich gebotenen Qualität erbracht werden.“
Nachweis über ihre Wirkung vorliegt. Die der Zufriedenheit der Patienten ist ein Weg, diesen Nachweis zu führen, und kommt damit zumindest für einen Teilbereich den Qualitätsanforderungen des Gesetzgebers nach. Außerdem können die erhobenen Daten dem Erhalt oder der Verbesserung der Wettbewerbsfähigkeit der Praxis dienen, sie liefern Einblicke in zwischenmenschliche Aspekte der Behandlung sowie die emotionale Haltung des Patienten gegenüber der Praxis (๑ Abb. 1). Einige medizinische Einrichtungen lassen sich

Qualität hat drei Dimensionen > Ein Überblick über die Struktur von Qualität unddenStellenwertderPatientenzufriedenheit innerhalb dieser soll den Umgang mit dem schwer fassbaren Begriff „Qualität“ erleichtern.

Für die Physiotherapiepraxis bietet sich an, das Konstrukt Qualität in sogenannte Dimensionen zu untergliedern. Das erleichtert die Kommunikation sowie die Umsetzung von Qualität im Alltag. Avedis Donabedian, ein amerikanischer Wissenschaftler, empfiehlt folgende Einteilung: Strukturqualität, Prozessqualität und Ergebnisqualität ( $\odot$ Abb. 2) [1].

Unter Strukturqualität versteht man unter anderem die Organisation der Einrichtung. Dazu gehören zum Beispiel TerminErfassung der Patientenperspektive und planung und Datenpflege, die Erreichbarkeit der Praxis und die technische Ausrüstung wie Behandlungsbänke und Materialien. Die Prozessqualität beschreibt, wie gut Therapieprozesse ablaufen. Dabei geht es zum Beispiel um die Zusammenarbeit mit Kollegen und Ärzten, um die Qualität von Untersuchungs- und Therapietechniken und um die Dokumentation von Behandlungsverläufen. Für die Ergebnisqualität werden die Erfolge der Behandlungen gemessen. Sie bildet damit die offensichtliche Verbesserung des Gesundheitszustandes ab. Hier lässt sich auch die Patientenzufriedenheit einordnen.

Mit einem Fragebogen die Ergebnisqualität messen > Die Kunst ist nun, das „Gute“ an einem Physiotherapeuten in Zahlen auszudrücken, das heißt, die Qualität muss irgendwie messbar gemacht werden.

Das Messen der Strukturqualität stellt keine größere Schwierigkeit dar. Es gibt meist klare Vorgaben und messbare Größen. Solche Vorgaben sind - auf die Physiotherapie bezogen - die Richtlinien vom Gesetzgeber, die zum Beispiel die Praxisausstattung betreffen. So müssen die Räumlichkeiten der Praxis in sich abgeschlossen sein, die Praxis sollte behindertengerecht zugänglich sein, ein Wartebereich mit ausreichend Sitzgelegenheit muss vorhanden sein usw. Die Krankenkassen lassen die vorhandene Strukturqualität prüfen; ohne deren sogenannte Abnahme wird die Kassenzulassung nicht erteilt. 

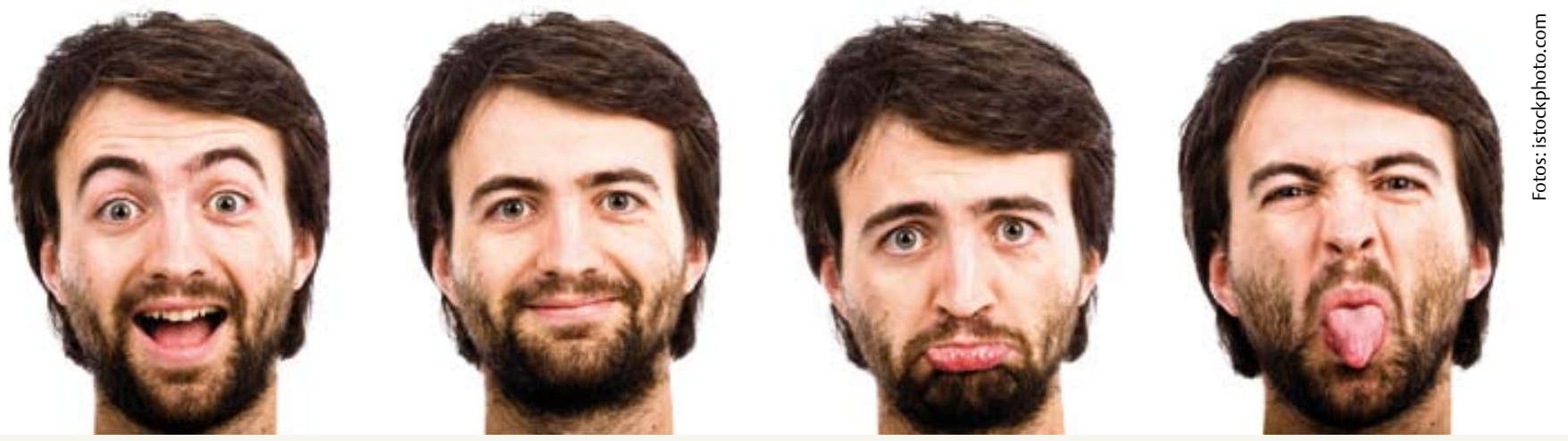

Abb. 1 Glücklich, zufrieden, unzufrieden oder gar verärgert:

Patienten können die Praxis in unterschiedlichster Stimmung verlassen.

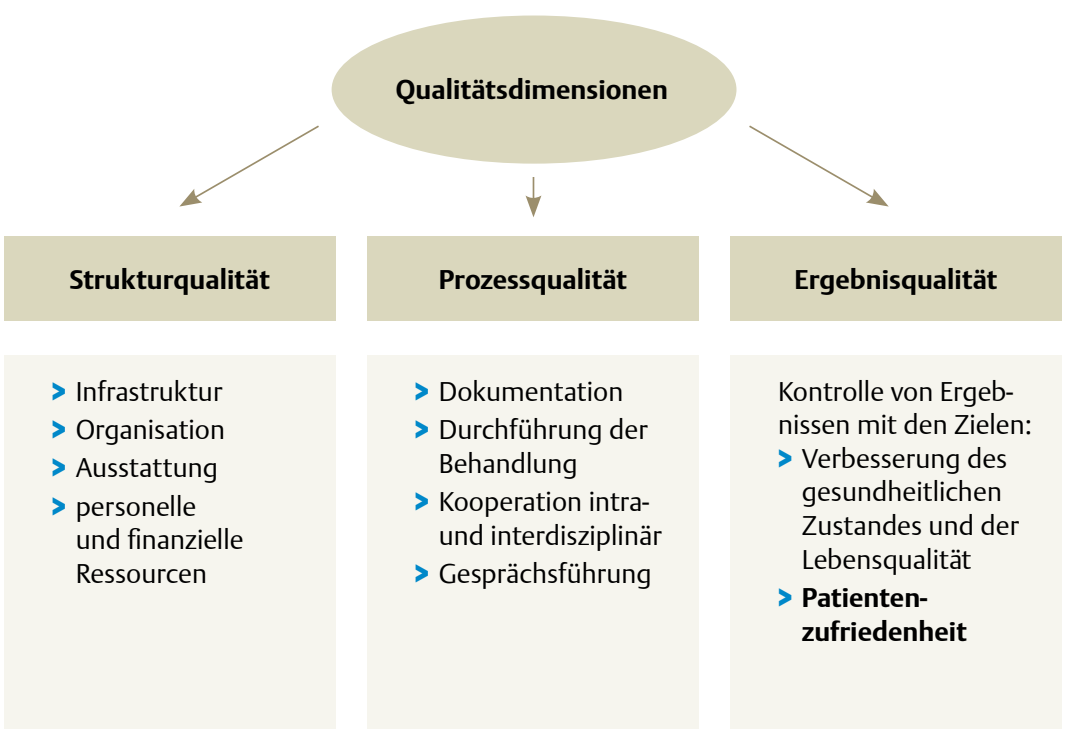

Abb. 2 Denkmodell zur den Dimensionen von Qualität. Sind Patienten zufrieden, schlägt sich das in der Dimension „Ergebnisqualität“ nieder.

Die Messung der Prozess- und Ergebnisqualität ist schwieriger durchzuführen, da hier mehr Gestaltungsfreiraum existiert. Die Heilmittelempfehlungen und Heilmittelrichtlinien schreiben zwar vor, dass Prozessqualität zu sichern ist, aber es gibt keine Vorgaben zur Art und Weise der Erfüllung. Es liegt also beim Einzelnen, wie er vorgeht [2]. Eine Möglichkeit, Teilprozesse und Teilergebnisse zu evaluieren, ist ein Fragebogen, der die Patientenzufriedenheit abfragt ( $\odot$ Abb. 3). Den Patienten oder Kunden in die Qualitätsbewertung mit einzubeziehen macht Sinn, da der Betroffene selbst am besten über die Behand- lung urteilen kann. Diese spezifische Kompetenz nützt dem Therapeuten, um eine Rückmeldung über Mängel und Ergebnisse der Behandlung herauszufinden und um dementsprechend den Fürsorgeprozess zu verbessern [3].

Patienten können Kunden werden > Wo liegt der Unterschied in der Bezeichnung Patient oder Kunde? Es ist nicht einerlei, denn hinter diesen Begriffen stecken unterschiedliche Bilder, wie der Therapeut seine Klientel wahrnimmt.

Seit einigen Jahren steigt die Erwartung der Patienten an die medizinische Versor-

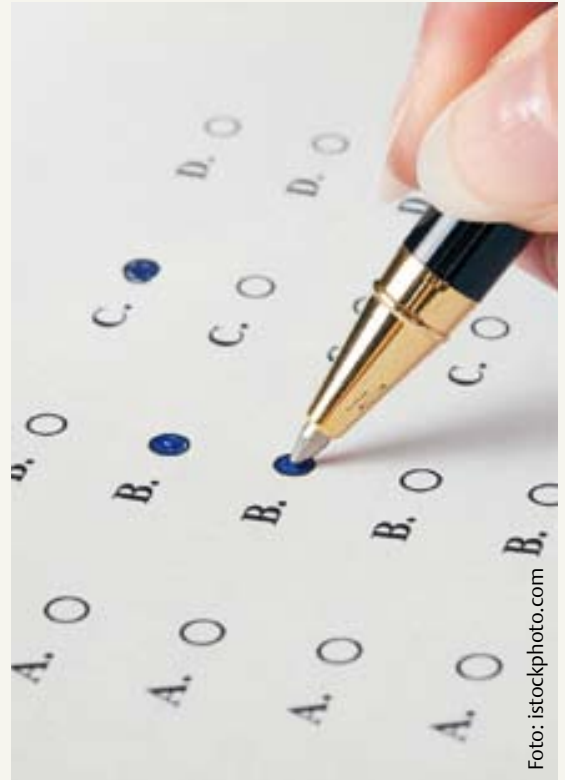

Abb. 3 Professionelles Qualitätsmanagement: Die Patientenzufriedenheit schriftlich abfragen

gung. Das liegt unter anderem daran, dass die Informationsbeschaffung über das Internet leichter geworden ist. Die Patienten fordern ihre Rechte ein. Dabei unterstützt sie die Bundesregierung: Helga Kühn-Mengel ist Patientenbeauftragte und soll insbesondere darauf hinwirken, dass die Belange der Patienten sowie deren Beratungs-, Informations- und Beteiligungsrechte berücksichtigt werden.

Des Weiteren vergrößert sich der Gesundheitsmarkt. Es geht nicht mehr nur um die reine Krankenversorgung, auch Prävention, Rehabilitation und die Verbesserung der Lebensqualität im Alltag werden 
wichtiger. Die Patienten nehmen das mit steigendem Gesundheitsbewusstsein wahr und sind bereit, für Leistungen auch selbst zu zahlen, also zum Kunden zu werden.

Und nicht zuletzt steigen die Kosten der medizinischen Versorgung, was die Krankenkassen aufgrund der älter werdenden Bevölkerung nicht mehr tragen können. Der Staat reagiert mit Ausgabenkontrollen wie zum Beispiel Selbstbeteiligungen oder Budgetierungen der Heilmittel.

Die Erwartungshaltung des Kunden > Durch diesen Systemwandel entwickelt der Patient eine größere persönliche Verantwortung. Die wiederum steigert seine Qualitätserwartungen an den Gesundheitsmarkt: Der Patient wird zum Kunden. Das erfordert bei Therapeuten ein Umdenken. Der Therapeut muss sich nun auf Dienstleistungen einstellen, die auf Kundenzufriedenheit abzielen. Experten diskutieren diese Sichtweise: Ist der Patient tatsächlich in der Lage, als Kranker über seine Behandlung mit zu entscheiden? Oder sollen Therapeuten als Experten allein entscheiden?

Der goldene Weg liegt irgendwo dazwischen. Je weniger fachkundig ein Patient ist und je komplexer sein Problem ist, desto stärker wird die Patientenrolle überwiegen. Demgegenüber wird eine fachlich aufgeklärte Person sehr viel stärker als Kunde auftreten, da sie die eigenen Bedürfnisse besser einzuschätzen weiß. Den Physiotherapeuten werden künftig immer mehr beide „Sorten“ begegnen: Der Patient einerseits, der Kunde mit erhöhter Erwartungshaltung andererseits.

Einflussfaktor gute Kommunikation > Um die Patientenzufriedenheit (und im weiteren Sinne die Kundenzufriedenheit) positiv zu beeinflussen, muss der Therapeut zunächst wissen, was dem Patienten innerhalb der Betreuung eigentlich wichtig ist. Neben Faktoren wie moderne Einrichtung, Sauberkeit und schnelle Terminvergabe stehen dabei hauptsächlich die sogenannten Softskills des Therapeuten im Vordergrund. Hierbei begutachtet der Patient die Interaktion zwischen Therapeut und ihm, ob er sich gut aufgehoben fühlt, ob der Therapeut Verständnis für ihn hat und ob eine zwischenmenschliche Basis vorhanden ist.
Ein wichtiger Faktor ist dabei die Kommunikationsfähigkeit des Therapeuten. Sie beinhaltet die Beziehungsebene und die informierende Ebene. Auf der Beziehungsebene werden die Emotionen des Patienten angesprochen, die andere Ebene hat einen inhaltlich-informierenden Charakter. Hier geht es darum, den Patienten möglichst objektiv über dessen gesundheitliches Problem und mögliche Folgen aufzuklären.

Kommunikation und Emotion > Der Patient kommt mit seiner individuellen Krankheits- und Lebensgeschichte zum Therapeuten. Er möchte von ihm Unterstützung im Umgang mit seiner Erkrankung. Neben dem Wunsch nach therapeutischer Intervention möchte er als Individuum wahrgenommen werden. Er erwartet emotionale Hilfe und Empathie beim Umgang mit seinen Beschwerden und Ängsten. Das Vertrauensverhältnis und der menschliche Umgang bieten den unerlässlichen Rahmen für eine erfolgreiche Behandlung. Ist diese „Chemie“ nicht gegeben, wird die Patientenzufriedenheit nicht gefördert.

\section{Wichtig für einen Patienten ist, dass der Therapeut ihm die Medizinersprache in verständliche Worte übersetzt.}

Kommunikation und Information > Patienten nutzen zunehmend das Internet als Informationsquelle. Sie können die Informationen allerdings oft nicht interpretieren, da ihnen das medizinische Wissen fehlt. Sie nehmen die Informationen mit zum Therapeuten und möchten sich die Hintergründe erklären lassen. Es ist dann notwendig, dass der Therapeut die kommunikative Fertigkeit besitzt, die Information dem Patienten in nicht medizinischer Sprache verständlich zu machen.

Einem Informationsdefizit auf Seiten des Patienten folgt eine schlechtere Compliance (= Therapiebereitschaft) und eine schlechtere Befindlichkeit [4]. Der Patient erwartet zum einen die fachkundige Übersetzung seiner oftmals fragmentarisch zusammengesetzten Informationen, zum anderen fungiert der Therapeut auch selbst als Informationsquelle.

Dabei spielt die verständliche Erklärung des Befundes, der abgeleiteten Behandlungsziele und der Maßnahmen eine wichtige Rolle. Wichtig für den Patienten sind außerdem die Erläuterung der Prognose und die Hilfe zur Selbsthilfe.

Sinnvolle Technik: Partizipative Entscheidungsfindung > Weiterhin zeigt sich, dass das Interaktionsmodell „Partizipative Entscheidungsfindung“ einen positiven Effekt auf die Patientenzufriedenheit und auch auf das Therapieergebnis hat. Es geht darum, dass Therapeuten und Patienten hinsichtlich der Behandlung gleichberechtigte Partner sind. Beide Parteien bringen ihre Entscheidungskriterien in den Therapieprozess ein und verantworten gemeinsam die getroffene Entscheidung. Der Therapeut bringt als Experte auf seinem Gebiet die wissenschaftlich fundierten Informationen ein, der Patient seine persönlichen Werte und Einstellungen. Anhand dessen legt man den Behandlungspfad gemeinsam fest (๑ physiopraxis 7-8/06, S. 59, „Partizipative Entscheidungsfindung“).

In vielen englischsprachigen Ländern wird dieses Modell schon eingesetzt. In Deutschland sind die Diskussionen noch recht jung. Wissenschaftliche Belege in Bezug auf die Physiotherapie fehlen zurzeit noch. Das Modell ist jedoch in der Arzt-Patient-Situation evidenzbasiert, was sich wahrscheinlich auf die physiotherapeutische Interaktion übertragen lässt.

Den subjektiven vom objektiven Behandlungserfolg unterscheiden > Die Beurteilung des Behandlungserfolgs durch den Patienten ist ein wichtiger Qualitätsindikator in der medizinischen Versorgung. Da der Patient in der Regel nicht über differenziertes medizinisches Wissen verfügt, ist diese Beurteilung als subjektiv einzustufen. Daneben kann man den Behandlungserfolg aber auch objektiv mit unterschiedlichen Assessments nachweisen. Die Patientenwahrnehmung kann vom objektiven Behandlungserfolg unabhängig sein, 
ja sogar im Widerspruch dazu stehen. Das heißt, eine objektiv erfolgreich verlaufene Behandlung wird vom Patienten nicht als solche wahrgenommen und beurteilt, wenn er subjektiv keine Verbesserung erkennt. Dessen muss man sich bewusst sein, wenn man die Patientenzufriedenheit erfasst. Dr. Oliver Ommen und seine Kollegen von der Universität Köln beschrieben diesen Zusammenhang zwischen subjektivem Behandlungserfolg und Zufriedenheit des Patienten in einer Studie [4].

Andere Einflüsse sind Geschlecht, Alter, Kontinuität und soziale Schicht $>$ In einer weiteren Studie zeigte sich, dass Frauen offensichtlich ein höheres Anspruchsniveau als Männer haben und demzufolge schwieriger zufriedenzustellen sind [5]. Und ein weiterer möglicher Einflussfaktor auf die Zufriedenheit stellt das Alter der Patienten dar. Es zeigte sich, dass ältere Patienten insgesamt höhere Zufriedenheitswerte hatten als jüngere [4].

Eine hohe Patientenzufriedenheit ist des Weiteren von einer gewissen Kontinuität abhängig, was den Therapeuten angeht. Wechsel in der Betreuung empfinden Patienten als nicht zufriedenstellend [6].

Es konnte außerdem nachgewiesen werden, dass Patienten aus niedrigen sozialen Schichten (das heißt mit geringer Bildung und niedrigem Einkommen) eher zufrieden sind als Menschen mit einem höheren sozioökonomischen Status [4].

Organisationsgrad nicht so wichtig > Bei Zufriedenheitsmessungen gibt es meist Fragen nach der Organisation im Sinne von übergeordneten Faktoren wie zum Beispiel Wartezeiten und Terminverschiebungen. Es zeigte sich, dass die Erwartungen der

\section{Als nicht so wichtig stufen Patienten organisatorische Dinge der Praxis ein.}

Patienten an solche organisatorischen Aspekte im Rahmen der Strukturqualität eher gering einzuschätzen sind [7]. Das heißt: Diese Aspekte sind nicht ausschlaggebend für die Zufriedenheit der Patienten. Im Vergleich mit den bereits genannten Einflussfaktoren spielen sie eine untergeordnete Rolle.

Soziale Erwünschtheit kann Ergebnisse eines Fragebogen verfälschen > Das Phänomen der sozialen Erwünschtheit beschreibt den Umstand, dass Patienten bei einer Befragung hohe Zufriedenheit äußern, obwohl sie in Wirklichkeit die Versorgung als (teilweise) unzulänglich empfinden [8]. Schuld daran sind die Angst vor persönlichen Nachteilen bei Kritik und die Angst vor dem Selbsteingeständnis, schlecht behandelt worden zu sein. Prof. Dr. Dr. Heiner Raspe und Kollegen bewerten Zufriedenheitsraten unter 75\% als „erhebliches Konfliktpotenzial“. Diese Rate bezieht sich auf eine Skala, deren Endpunkte mit $0 \%$ (= völlig unzufrieden) und $(100 \%=$ völlig zufrieden $)$ markiert sind. Der Konflikt (bzw. die Unzufriedenheit) entsteht durch die Differenz zwischen den Ansprüchen der Patienten und dem, was ihm in der Praxis geboten wird. Und selbst bei einer vordergründig „guten“ Zufriedenheit von $75 \%$ bewertet Raspe den Konflikt als deutlich vorhanden, weil er das Phäno- men der sozialen Erwünschtheit „mit reinkorrigiert“. Die Erwartungen des Patienten sind dann weit weniger erfüllt, als dessen Bewertung vordergründig glauben macht.

Zu wenig Kommunikationstraining in Aus- und Fortbildung > Betrachtet man zusammenfassend die Einflüsse auf die Zufriedenheit von Patienten, stellt sich als besonderes Qualitätsmerkmal die Kommunikationsfähigkeit des Therapeuten heraus. Dieser Aspekt wird, gemessen an seiner Bedeutung, in der Aus- und Fortbildung zu wenig berücksichtigt, da hier der Schwerpunkt auf die Vermittlung fachlicher Fertigkeiten gelegt wird. Die Fähigkeit, über Kommunikation eine gute Beziehung zum Patienten zu schaffen und damit die Patientenzufriedenheit positiv zu beeinflussen, gehört in den Werkzeugkoffer eines jeden Therapeuten.

Wünschenswert ist außerdem, dass das Thema Qualitätsmanagement in der Ausbildung implementiert wird. Der Aspekt Patientenzufriedenheit ist Teil des Qualitätsanspruchs eines Physiotherapeuten. Auf dem Weg, ein guter Physiotherapeut zu werden, ist also die Berücksichtigung dessen unumgänglich.

Karen Friedel

\section{$\Rightarrow$ Die Zahlen in eckigen Klammern weisen auf das Literaturverzeichnis hin. Dieses finden Sie unter www.thieme.de/physio- online > "physiopraxis“ > "Literatur" > „physiopraxis 6/08“.}

$\rightarrow$ In der nächsten physiopraxis gibt es den zweiten Teil des Artikels. Darin stellt die Autorin den „Patientenfragebogen zur Qualität gesundheitlicher Versorgung Physiotherapie (PQV-PT)“ vor.

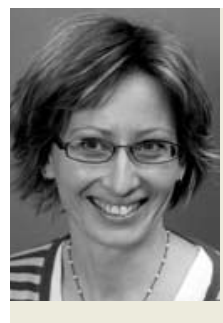

Karen Friedel ist seit 1995 Physiotherapeutin. 2007 hat sie den Bachelor an der niederländischen Hochschule Thim van der Laan, Nieuwegein, erworben. Jetzt ist sie fachliche Leiterin eines Rückenzentrums in Köln und Dozentin an den Döpferschulen Köln sowie an der Hochschule Thim van der Laan.
Sie von $A$ bis $Z$ unterstützen, zum Beispiel mit fertigen Fragebögen, mit deren statistischer Analyse sowie einem Vergleich mit der Patientenzufriedenheit in anderen unter www.thieme.de/ physioonline mit dem Stichwort „Patientenbefragung“. 